\title{
Overview of diffuse optical tomography and its clinical applications
}

Yoko Hoshi

Yukio Yamada 


\title{
Overview of diffuse optical tomography and its clinical applications
}

\author{
Yoko Hoshia,* and Yukio Yamada ${ }^{\mathrm{b}}$

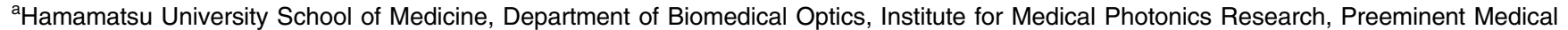 \\ Photonics Education and Research Center, 1-20-1 Handayama, Higashi-ku, Hamamatsu 431-3192, Japan \\ bUniversity of Electro-Communications, Brain Science Inspired Life Support Research Center, 1-5-1 Chofugaoka, Chofu, Tokyo 182-8585, Japan
}

\begin{abstract}
Near-infrared diffuse optical tomography (DOT), one of the most sophisticated optical imaging techniques for observations through biological tissue, allows 3-D quantitative imaging of optical properties, which include functional and anatomical information. With DOT, it is expected to be possible to overcome the limitations of conventional near-infrared spectroscopy (NIRS) as well as offering the potential for diagnostic optical imaging. However, DOT has been under development for more than 30 years, and the difficulties in development are attributed to the fact that light is strongly scattered and that diffusive photons are used for the image reconstruction. The DOT algorithm is based on the techniques of inverse problems. The radiative transfer equation accurately describes photon propagation in biological tissue, while, because of its high computation load, the diffusion equation (DE) is often used as the forward model. However, the DE is invalid in low-scattering and/or highly absorbing regions and in the vicinity of light sources. The inverse problem is inherently ill-posed and highly undetermined. Here, we first summarize NIRS and then describe various approaches in the efforts to develop accurate and efficient DOT algorithms and present some examples of clinical applications. Finally, we discuss the future prospects of DOT. (C) The Authors. Published by SPIE under a Creative Commons Attribution 3.0 Unported License. Distribution or reproduction of this work in whole or in part requires full attribution of the original publication, including its DOI. [DOI: 10.1117/1.JBO.21.9.091312]
\end{abstract}

Keywords: radiative transfer equation; diffusion equation; optical properties; model-based iterative image reconstruction. Paper 160068SSVR received Feb. 1, 2016; accepted for publication Jun. 13, 2016; published online Jul. 13, 2016.

\section{Introduction}

Jöbsis ${ }^{1}$ first reported biomedical applications of near-infrared spectroscopy (NIRS); NIRS was originally designed for clinical monitoring, and it has also become a useful tool for neuroimaging studies with the so-called functional NIRS (fNIRS). ${ }^{2-5}$ (In this context, the term "NIRS" is sometimes used to express the optical method for studying brain functions apart from the original meaning of NIRS, causing some confusions in the community. In this article, the authors keep the original meaning of NIRS.) In parallel to these developments, optical computed tomography (CT) using near-infrared (NIR) light has been and is still being developed. Optical CT is a technique to reconstruct images of optical properties, including the absorption $\left(\mu_{\mathrm{a}}\right)$ and reduced scattering coefficients $\left(\mu_{\mathrm{s}}^{\prime}\right)$, within highly scattering media from measurements of the light propagation at the tissue boundary. $^{6-9}$

The photons emerging from a thin turbid medium consists of ballistic (coherent), snake (quasicoherent), and diffusive (incoherent) components. The ballistic, snake, and diffusive components refer to photons traveling along the straight-line path without being scattered, photons traveling along quasistraight-line paths with experiencing only a few scattering events, and photons traveling along zig-zag paths with being scattered many times in all directions, respectively. ${ }^{10,11}$ When the thickness of biological tissue is sufficiently thin (less than a few millimeters) so that ballistic and/or snake photons can reach a detector, ${ }^{10,11}$ a conventional image reconstruction algorithm for X-ray CT can be applied to optical CT. ${ }^{12-14}$ However, in

*Address all correspondence to: Yoko Hoshi, E-mail: yhoshi@ hama-med.ac.jp the case of thicker tissue, the ballistic and snake components of the transmitted light are extremely rare due to the strong light scattering, and here, the diffusive component is exclusively detected at the tissue boundary. Because of this image reconstruction algorithms utilizing diffusive photons were proposed in the early $1990 \mathrm{~s},{ }^{15-17}$ and a variety of algorithms for diffuse optical tomography (DOT) based on the technique of the inverse problem has been proposed. ${ }^{18-20}$ However, DOT is still a technique that is under development and not fully ready for full-scale application.

Today, DOT is often confused with optical topography based on the spatial interpolation method (see below) with multichannel NIRS instruments, in general, continuous wave (CW) instruments (see Sec. 2.3). Optical topography, which is typically applied in functional brain mapping, is roughly divided into two categories on the basis of the selected image reconstruction approach. The first is to generate topographic images using a spatial interpolation method for the changes in NIRS signals at all measurement points without depth discrimination. ${ }^{21,22}$ This approach is commonly used in fNIRS studies, although the lateral spatial resolution is limited to the source-detector distance. $^{23,24}$ Since the depth resolution is not considered, i.e., it is assumed that all changes are occurring in a single layer, these topographic images are inherently influenced by variations in the depth direction, such as the presence of the cerebrospinal fluid $(\mathrm{CSF})^{25}$ and changes in the optical properties of the extracerebral tissue including skin blood flow changes. ${ }^{26,27}$ These problems could be solved by DOT.

The other category of optical topography is to reconstruct images based on the techniques of inverse problems using data acquired at multiple source-detector distances. ${ }^{28,29}$ This 
approach improves the spatial resolution and provides some depth discrimination. A research group at the University College London (UCL) argued that topography of this type be distinguished from optical tomography based on the aims of the experiments involved. ${ }^{30}$ When a three-dimensional (3-D) volume is intended to be reconstructed from light detected at the surface opposite to the source position in the transmittance mode, they propose to term it optical tomography. If the intention is to measure activity in a small portion of the head with limited depth discrimination and high temporal resolution, the term they propose is optical topography. In the report here, we will consider the topography of this type as being DOT, based on the classification of the image reconstruction algorithms involved, which has since been also adopted by the UCL group.

In this paper, we first describe the basic theory of NIRS and then review the image reconstruction algorithms and the clinical applications. Finally, future prospects for DOT will be discussed.

\section{Basic Principles of Near-Infrared Spectroscopy}

\subsection{Near-Infrared Spectroscopy}

NIRS, utilizing light in the NIR region, wavelength range approximately from 700 to $2500 \mathrm{~nm}$, is a noninvasive technique for analysis of various substances including agricultural products and food. Biological tissue is relatively transparent for light in the wavelength range from 650 to $950 \mathrm{~nm},{ }^{31}$ the so-called optical window, where absorption spectra vary with the oxygenation-deoxygenation states of hemoglobin $(\mathrm{Hb})$ and myoglobin and the redox state of cytochrome c oxidase. These spectroscopic characteristics were the background for the biomedical applications of NIRS: light sources emit NIR light on the body surface, and the light propagates through biological tissue and the reflected light emerging a few centimeters from the incident position is detected. This kind of NIRS has typically been applied to measure concentration changes in cerebral $\mathrm{Hb}$ qualitatively, although organs and tissue other than the brain, such as muscle tissue, ${ }^{32,33}$ and the redox state of cytochrome c oxidase ${ }^{34,35}$ have also been measured with NIRS.

Presently, NIRS has become widely applied in a variety of neuroimaging studies as well as in clinical monitoring of tissue oxygenation. ${ }^{36}$ The introduction of multichannel NIRS instruments, which are mainly used for optical topography, is facilitating neuroimaging studies using light, while difficulties in the selective and quantitative measurements of cerebral $\mathrm{Hb}$, a central issue in the NIRS field, remain to be overcome.

\subsection{Modeling Light Propagation in Biological Tissue}

The main interaction between light and biological tissue is absorption and scattering when the wave nature of light is neglected. Biological tissue, in which scattering is generally dominant over absorption, exhibits a highly anisotropic forward scattering, ${ }^{37,38}$ and light propagation in biological tissue is characterized by the optical properties of absorption, scattering, scattering anisotropy, and refractive indexes. Understanding light propagation is crucial for developing therapeutic and diagnostic optical techniques and for the quantitative analysis of measurements. ${ }^{39,40}$ The radiative transfer equation (RTE) and diffusion equation (DE) are the most commonly used as light propagation models.

\subsubsection{Radiative transfer equation}

It is widely accepted that the RTE, which is based on the law of conservation of energy for light propagation through a volume element of a medium with absorbers and scatterers, correctly describes light propagation in biological tissue. ${ }^{41-44}$ The timedomain (TD) RTE is expressed as

$$
\begin{aligned}
& {\left[\frac{\partial}{v(\mathbf{r}) \partial t}+\boldsymbol{\Omega} \cdot \nabla+\mu_{\mathrm{a}}(\mathbf{r})+\mu_{\mathrm{s}}(r)\right] I(\mathbf{r}, \boldsymbol{\Omega}, t)} \\
& \quad=\mu_{\mathrm{s}}(\mathbf{r}) \int_{4 \pi} \mathrm{d} \boldsymbol{\Omega}^{\prime} P\left(\mathbf{r}, \boldsymbol{\Omega} \cdot \boldsymbol{\Omega}^{\prime}\right) I(\mathbf{r}, \boldsymbol{\Omega}, t)+q(\mathbf{r}, \boldsymbol{\Omega}, t),
\end{aligned}
$$

where $I(\mathbf{r}, \boldsymbol{\Omega}, t)$ is the energy radiance (the light intensity) as a function of the position $\mathbf{r}=(x, y, z)$, angular direction $\boldsymbol{\Omega}=(\sin \theta \cos \phi, \sin \theta \sin \phi, \cos \theta)$ with the zenith angle $\theta$ and azimuth angle $\phi$, and time $t$. The $\mu_{\mathrm{a}}(\mathbf{r})$ and $\mu_{\mathrm{s}}(\mathbf{r})$ terms are the absorption and scattering coefficients, respectively, $v$ is the velocity of light in a turbid medium, and $q(\mathbf{r}, \boldsymbol{\Omega}, t)$ is the light source. Further, $P\left(\mathbf{r}, \boldsymbol{\Omega} \cdot \boldsymbol{\Omega}^{\prime}\right)$ is the scattering phase function that describes the probability that a photon with the direction $\boldsymbol{\Omega}^{\prime}$ is scattered to the direction $\boldsymbol{\Omega}$ during a scattering event. The phase function $P$ is normalized with

$$
\int_{4 \pi} \mathrm{d} \mathbf{\Omega}^{\prime} P\left(\mathbf{r}, \boldsymbol{\Omega} \cdot \mathbf{\Omega}^{\prime}\right)=2 \pi \int_{-1}^{1} P(\cos \theta) \mathrm{d} \cos \theta=1 .
$$

The Henyey-Greenstein function [Eq. (3)] is commonly employed as the scattering phase function ${ }^{45}$

$$
P\left(\mathbf{r}, \boldsymbol{\Omega} \cdot \mathbf{\Omega}^{\prime}\right) \quad=\frac{1}{4 \pi} \frac{1-g(\mathbf{r})^{2}}{\left[1+g(\mathbf{r})^{2}-2 g \Omega \cdot \Omega^{\prime}\right]^{\frac{3}{2}}},
$$

where $g(\mathbf{r})$ is the anisotropic factor ranging from -1 (complete backscattering) via 0 (isotropic scattering) to +1 (complete forward scattering).

\subsubsection{Diffusion equation}

The RTE is an integro-differential equation, and it is difficult to solve it analytically; also, the computation load for numerical solutions is extremely heavy. Alternatively, light propagation in biological tissue is commonly modeled by the DE based on the assumption that the radiance in an optically thick medium, in which multiple scattering occurs, is almost isotropic. The DE is derived from a diffusion approximation to the RTE in which the intensity $I(\mathbf{r}, \boldsymbol{\Omega}, t)$ is approximated by the first two terms of a series expansion in spherical harmonics ( $P_{1}$ approximation), the temporal variation in the flux vector $\left[\mathbf{J}(\mathbf{r}, t)=\int_{4 \pi} \boldsymbol{\Omega} I(\mathbf{r}, \boldsymbol{\Omega}, t) \mathrm{d} \boldsymbol{\Omega}\right]$ is assumed to be negligible, and the light source is isotropic. ${ }^{7}$ The time domain DE is expressed as

$$
\left[\frac{\partial}{v(\mathbf{r}) \partial t}-D \nabla^{2}+\mu_{\mathrm{a}}(\mathbf{r})\right] \Phi(\mathbf{r}, t)=q(\mathbf{r}, t),
$$

where $\Phi(\mathbf{r}, t)$ is the fluence rate given by $\int_{4 \pi} \mathrm{d} \boldsymbol{\Omega} I(\mathbf{r}, \boldsymbol{\Omega}, t), D$ is the diffusion coefficient given by $\left[3(1-g) \mu_{\mathrm{s}}(\mathbf{r})\right]^{-1}$ for the time domain, ${ }^{46,47}$ and $q(\mathbf{r}, t)$ is the isotropic source given by $\int_{4 \pi} \mathrm{d} \boldsymbol{\Omega} q(\mathbf{r}, \boldsymbol{\Omega}, t)$. The term $(1-g) \mu_{\mathrm{s}}=\mu_{\mathrm{s}}^{\prime}$ is the reduced scattering coefficient. 


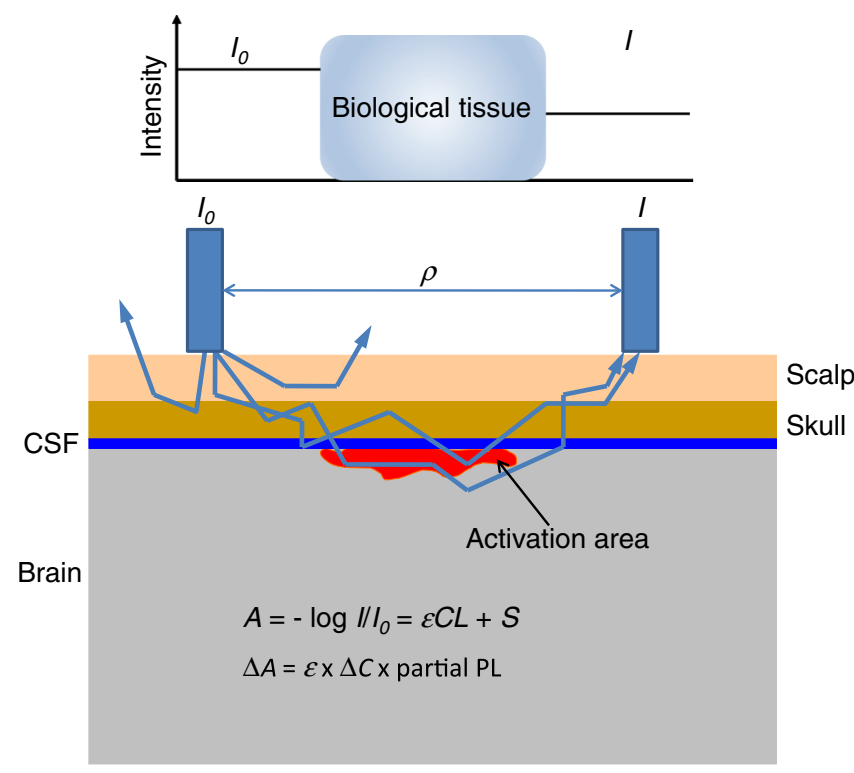

Fig. $1 \mathrm{CW}$ measurement in a brain model and the MBLL.

\subsection{Types of Near-Infrared Spectroscopy Measurements}

A wide range of NIRS instruments, which are able to perform various types of measurement, have been developed. Figures 1-3 show three representative types of measurements: (1) CW measurement (steady-state domain measurement), (2) TD measurement, and (3) frequency-domain (FD) measurement.

\subsubsection{Continuous wave measurements}

In CW measurement, the light source emits light at a constant intensity into the tissue and the transmitted and back scattered light intensities are measured (Fig. 1). In general, commonly available $\mathrm{CW}$ instruments analyze the transmitted light based on the modified Beer-Lambert law (MBLL). ${ }^{48}$ The MBLL is expressed as

$A=-\log I / I_{0}=\varepsilon C L+S$

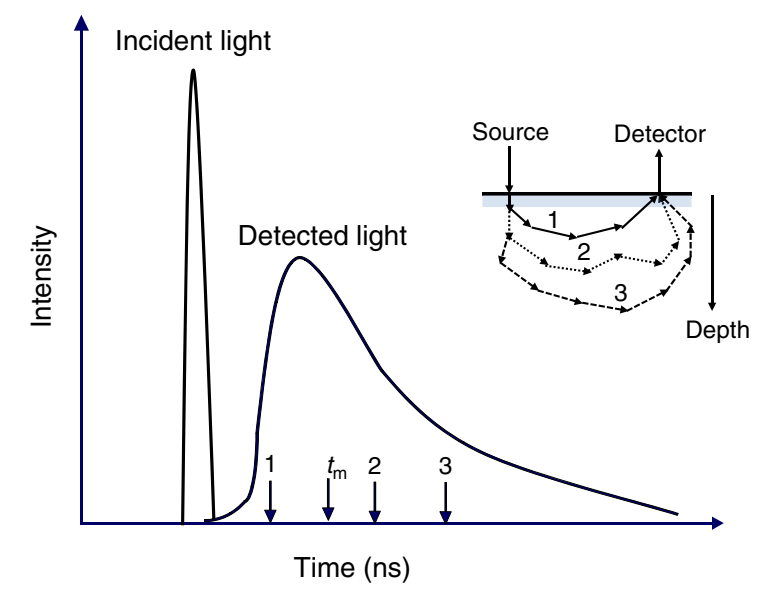

Fig. 2 TD measurement. Photons traveling through each path (1 to 3 ) shown in the inserted figure are detected at corresponding time (1 to 3). $t_{\mathrm{m}}$, mean time of flight.

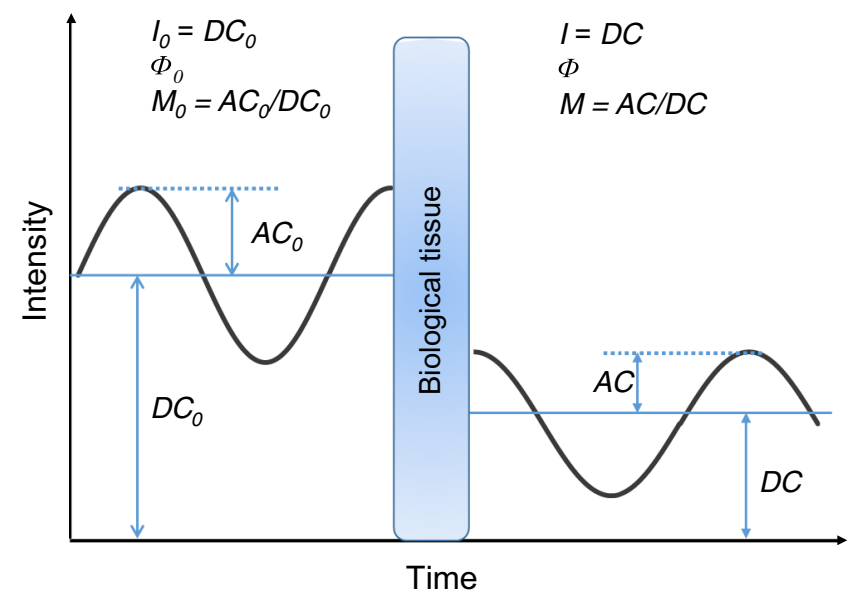

Fig. 3 FD measurement.

where $A$ is the attenuation measured in optical density, $I$ and $I_{0}$ are the intensities of the detected and incident light, $\varepsilon$ is the molar absorption coefficient, $C$ is the concentration of chromophore (e.g., $\mathrm{Hb}$ ), and $L$ is the mean length of the optical path, also known as the "mean total pathlength" (t-PL), and $S$ denotes the optical attenuation mainly due to scattering. However, CW instruments cannot measure the optical pathlength, with the result that instruments of this type do not provide absolute values of concentration changes, and the NIRS signal is expressed as the product of concentration change and optical pathlength. To overcome the problem with quantification, TD (timeresolved spectroscopy, TRS) ${ }^{48,49}$ and FD measurements (frequency-domain spectroscopy), ${ }^{50,51}$ which can determine the t-PL have been developed. However, functional brain activation is accompanied by localized $\mathrm{Hb}$ concentration changes within the cerebral tissue, which requires the determination of mean partial optical path lengths (p-PL) in the cerebral tissue for the quantification, something that is not feasible at present. Since both the t-PL and p-PL vary with the region of measurement, the amplitudes of NIRS signals for different regions reflect both concentration changes in the cerebral $\mathrm{Hb}$ and also other phenomena in the tissue involved.

\subsubsection{Time-domain measurements}

In TD measurements, the tissue is irradiated by ultrashort (picosecond order) laser pulses, and the intensity of the emerging light is recorded over time to show a temporal point spread function (TPSF) with picosecond resolution (Fig. 2). The mean t-PL is determined by multiplying the light speed in the media by the mean transit time of the scattered photons, which is calculated with the TPSF. The TRS data carry information about depthdependent attenuation based on the correlation of the detection time to the penetration depth of photons. Changes in cerebral $\mathrm{Hb}$ concentrations can be more selectively and quantitatively determined by analyzing the TPSF, as the TPSF contains information about the optical properties of the media, i.e., the absorption $\left(\mu_{\mathrm{a}}\right)$ and reduced scattering $\left(\mu_{\mathrm{s}}^{\prime}\right)$ coefficients.

Several different methods for analyzing the TPSF have been proposed. ${ }^{52-55}$ One method is a curve-fitting method based on the diffusion equation (the DE, DE-fit method) (see Sec. 2.2). In this method, optical properties are determined by fitting the TPSF, the temporal probability function for all detected photons, obtained from the analytical solution to the DE to the measured TPSF with the assumption that the human head is a semi-infinite 
homogeneous medium. Here it has been reported that the estimated $\mu_{\mathrm{a}}$ by the DE-fit method is very similar to the deeperlayer $\mu_{\mathrm{a}}$ under conditions where the $\mu_{\mathrm{a}}$ of the upper layer is larger than that of the lower layer in two-layered slab models. ${ }^{56}$ We have also confirmed that the DE-fit method is more sensitive to $\mu_{\mathrm{a}}$ changes in cerebral tissue by measuring adult heads with TRS during carotid endoarterectomy. ${ }^{57}$ This is explained by the fact that the later (falling) part of the TPSF, which includes more photons traveling through the cerebral tissue, is critical for the determination of the $\mu_{\mathrm{a}}$ in the fitting process. ${ }^{58}$ Overall, this allows changes in cerebral $\mathrm{Hb}$ concentrations to be more selectively measured by TRS than with CW measurements, although the selective and quantitative accuracies are not perfect.

\subsubsection{Frequency-domain measurements}

In FD measurements shown in Fig. 3, the light source is sinusoidally intensity-modulated at radio frequencies, in general, between 100 and $1000 \mathrm{MHz},{ }^{59}$ and the light intensity $(I$, the DC component), its phase shift $(\Phi)$, and modulation depth $(M)$, the ratio of $\mathrm{AC}$ (the amplitude of the intensity oscillations, the $\mathrm{AC}$ component) to $\mathrm{DC}$ with respect to the incident light are measured. The information obtained with TPSF through TD measurements can be obtained by FD measurements, the relation between the time and frequency information being the Fourier transform; however, measuring $I, \Phi$, and $M$ for all frequencies (from DC to $10 \mathrm{GHz}$, when TD measurements are made with a resolution of $10 \mathrm{ps}$ ) are required to obtain the TD data. ${ }^{60}$ It has been demonstrated that for typical tissue, and at frequencies below $200 \mathrm{MHz}, \Phi$ is linearly related to the mean t-PL. ${ }^{60}$ Based on analytical solutions of the frequency domain DE for a homogeneous infinite medium, ${ }^{61,62}$ numerical expressions including $\mu_{\mathrm{a}}$ and $\mu_{\mathrm{s}}^{\prime}$ in the FD measurement parameters have been derived, enabling experimentally based estimates of optical properties. ${ }^{63,64}$

\section{Diffuse Optical Tomography Algorithms}

\subsection{General Concept of Diffuse Optical Tomography}

DOT recovers 3-D distributions of the optical properties from multiple boundary measurements. With $\mu_{\mathrm{a}}=2.303 \varepsilon C$ (molar absorption coefficient $\mathrm{x}$ concentration), and unlike conventional
NIRS, DOT could enable a quantitative determination of regional cerebral $\mathrm{Hb}$ without contamination of signals arising from extracerebral tissue (Fig. 4). The DOT algorithm essentially consists of two parts: one is a forward model to calculate the light propagation and the resultant outward re-emissions at the boundary of the tissue, typically based on the DE or the RTE. The other is an inverse model searching for the distribution of optical properties.

The DOT image reconstruction can be approximately divided into two kinds: one is a linearization approach and another is a nonlinear iterative approach. The linearization approaches use Born or Rytov approximations, and the linearization is developed from an analytical (Green's function) solution to the DE for a homogeneous semi-infinite background ${ }^{54,65}$ with assumed known optical properties. Linearization approaches were employed to earlier DOT algorithms, ${ }^{66,67}$ but as they cannot correctly predict large changes in the optical properties because of the limitations of the Born or Rytov approximations, ${ }^{68}$ nonlinear iterative approaches have also been investigated. Recently, however, DOT based on linearization approaches has attracted attention in neuroimaging studies. Here, brain activity-related $\mu_{\mathrm{a}}$ changes are very small, the Born and Rytov approximations hold, and high-quality images are provided by high-density DOT systems (CW instruments with spatially overlapping multidistance source-detector arrangements). ${ }^{69,70}$ This approach reconstructs only qualitative images of measured changes, which is, however, sufficient for functional neuroimaging studies. In clinical use, quantitative images of steady-state $\mathrm{Hb}$ are further useful with diagnostic optical imaging, and DOT based on the nonlinear approaches have been developed to enable this. Implementation of DOT with both approaches is possible with CW, TD, and FD measurements, where the TD measurements provide more of the information required for image reconstruction.

\subsection{Linearization Approaches}

In linearization approaches, the relationship between the optical properties and measurements of the diffusive light is linearized; here, the forward problem is expressed as

$\mathbf{y}=\mathbf{A x}$. (a)

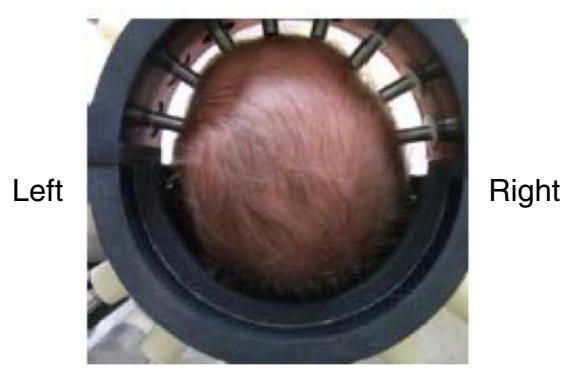

(b)

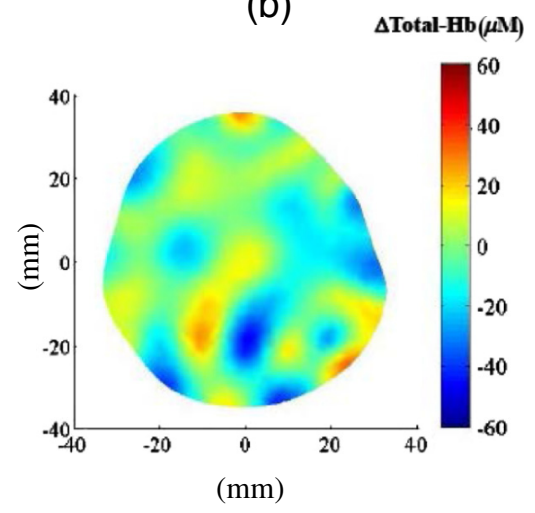

Fig. 4 DOT image of total $\mathrm{Hb}$ concentrations of the brain in an extremely low birth weight infant under hypocapnic conditions. (a) Sixteen coaxial optical fibers, consisting of a single fiber for illumination in the center and outer bundle fibers for detection, were placed on the scalp of the infant. (b) Image reconstruction was performed by using the DE-based algorithm. The axial image shows the total Hb concentration changes cause by hypocapnia in comparison with normocapnic conditions. (From Ref. 123, with permission.) 
The vectors $\mathbf{y}$ and $\mathbf{x}$ are a set of measurements and perturbed optical properties $\left(\mu_{\mathrm{a}}\right.$ and $\left.\mu_{\mathrm{s}}^{\prime}\right)$ in discrete volume elements (voxels), respectively. The matrix $\mathbf{A}$ is the Jacobian or sensitivity matrix. The vector $\mathbf{x}$ can be obtained from the vector $\mathbf{y}$ through inversion of matrix $\mathbf{A}$. Since, however, the number of unknowns is larger than the number of measurements, regularization methods are usually used to solve inverse problems. While various inverse solutions have been developed, a widely used approach is the single step solution with Tikhonov regularization and a Moore-Penrose generalized inverse. ${ }^{28,69-71}$

The main issues of single step algorithms are the difficulty in depth localization and low-image quality. ${ }^{72}$ To solve these issues, spatial variant regularization ${ }^{73,74}$ and high-density source-detector arrangements ${ }^{68}$ have been used. Further, recent studies have proposed a variety of advanced methods, such as methods using sparse regularization ${ }^{75,76}$ and a Bayesian approach, ${ }^{72,77-79}$ which require iterative processes. To reduce calculation times and avoid Born and Rytov approximation errors, a noniterative linear approach based on compressed sensing theory has also been developed. ${ }^{80,81}$

\subsection{Nonlinear Iterative Approaches}

\subsubsection{Model-based iterative image reconstruction}

In general, the DE is used as the forward model and it is typically solved numerically by the use of the finite element method. A number of image reconstruction methods have been reported, but the most widely used is based on the iterative optimization scheme, in which the distribution of optical properties is searched for by minimizing the differences between the calculated and the measured data (model-based iterative image reconstruction, ${ }^{82,83}$ Fig. 5).

There are two approaches to this optimization problem: one is based on a perturbation method that requires repeated construction and inversion of a large full Jacobian matrix (Newton-like methods). ${ }^{84,85}$ The other is an approach using gradient-based reconstruction techniques, such as the conjugated gradient method. ${ }^{86,87}$ In this approach, an objective function describes the difference between the calculated and measured data; the gradient of the objective function in a line minimization is used for the updating. This approach does not require inversion of the Jacobian matrix, offering a reduction in computation load, while the convergence is slow. The Newton-like methods, in which the convergence is faster, were the most commonly applied image reconstruction method in the 1990s, while they

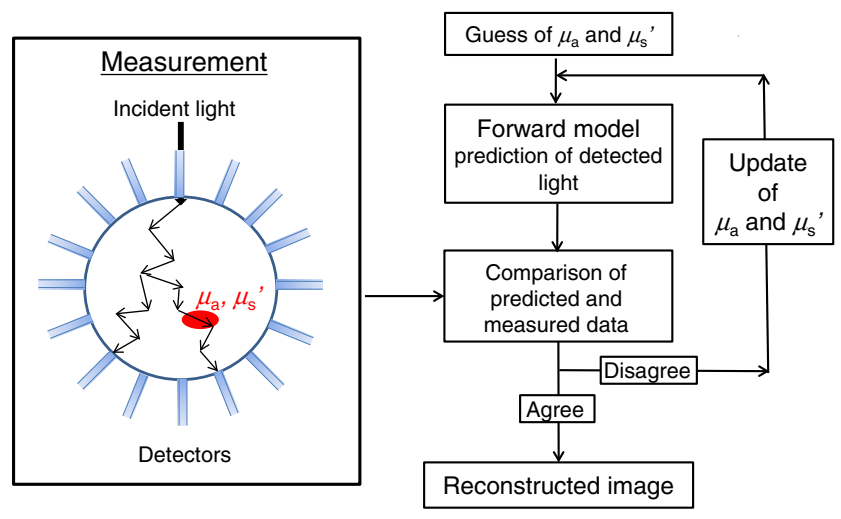

Fig. 5 Model-based iterative image reconstruction scheme. are computationally expensive and need to meet the assumption that changes in optical properties are small. As an alternative, a gradient-based iterative image reconstruction scheme has been proposed. However, these optimization problems are inherently ill-posed, and regularization terms generally have to be added.

\subsubsection{Data types}

As detailed in Sec. 3.1, TD measurements provide more data that can be used for image reconstruction than CW and FD measurements. With the TD data, the temporal profile of the detected light (Fig. 2), the (1) integral intensity, (2) $n$ 'th temporal moment, (3) $n$ 'th central moment, (4) normalized Laplace transform, and (5) Mellin-Laplace are derived. ${ }^{88}$ It has been demonstrated that image reconstruction using all of the timeresolved data improves image quality over that reconstructed with a single or combined moments, ${ }^{89}$ but it is still not conclusively established which data should be used to obtain high quality images. ${ }^{90,91}$

\subsection{Hybrid Model Based on the Radiative Transfer Equation and the Diffusion Equation}

The DE is widely used as the forward model, but it becomes invalid in low-scattering regions (e.g., CSF space and trachea), highly absorbing regions (e.g., hematoma), and in the vicinity of light sources, where the diffusion approximation does not hold, ${ }^{92,93}$ and under such disadvantageous conditions, RTEbased DOT algorithms ${ }^{94,95}$ are required. Several studies on the RTE-based forward model have been reported. ${ }^{96-98}$ Typically, the RTE is solved numerically, with only a few analytical solutions reported. ${ }^{99,100}$ One of the most common methods for solving the RTE is the discrete-ordinate method, which is compatible with finite difference, finite volume, or finite element schemes. $^{97,101}$

Even with current advanced computer technology, however, the heavy computational load is still the biggest drawback with the RTE. A variety of fast solvers of the RTE have been proposed, ${ }^{102-105}$ and among these a hybrid model-based the RTE and DE is one of the most promising approaches. The hybrid model is based on the idea that the DE is an accurate model for measurements far from a light source. This model has been proposed in frequency domains ${ }^{102,106,107}$ where a medium is divided into RTE and DE regions by a crossover interface that is determined by the crossover length beyond which the DA is valid. The concept of the hybrid model in the steady state has recently been extended into the time domain. ${ }^{93,108}$ Here, light propagation in homogeneous two-dimensional rectangular media $(3.2 \mathrm{~cm} \times 4.0 \mathrm{~cm})$, like with the optical properties shown in Table 1 , is estimated by using three numerical models, the DE, the RTE with $g=0.8$, and the RTE with $g=0$. Figure $6(\mathrm{a})^{93}$

Table 1 Optical properties of the numerical models.

\begin{tabular}{lccccc} 
& $\mu_{\mathrm{a}}\left(\mathrm{cm}^{-1}\right)$ & $\mu_{\mathrm{s}}\left(\mathrm{cm}^{-1}\right)$ & $\mu_{\mathrm{s}}^{\prime}\left(\mathrm{cm}^{-1}\right)$ & $g$ & $n$ \\
\hline RTE & 0.35 & 11.6 & 11.6 & 0.0 & 1.56 \\
RTE & 0.35 & 58.0 & 11.6 & 0.8 & 1.56 \\
DE & 0.35 & & 11.6 & & 1.56 \\
\hline
\end{tabular}




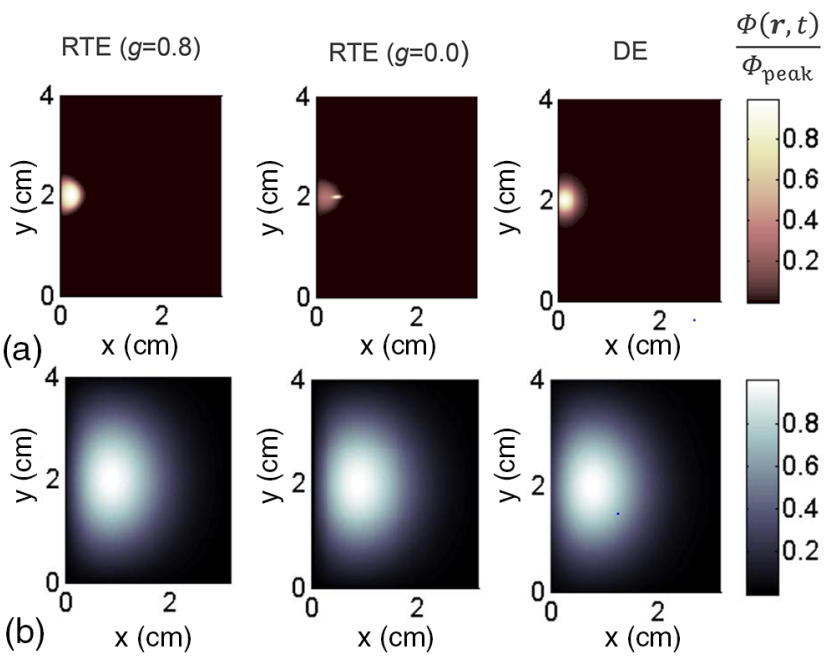

Fig. 6 Spatial distributions of fluence rate $(\Phi)$ normalized by its maximum value $\left(\Phi_{\text {peak }}\right)$ at a given time (a) $t=20$ ps and (b) 500 ps based on the RTE with $g=0.8$ and the anisotropic source, the RTE with $g=0.0$ and anisotropic source, and the DE. (From Ref. 91, with permission).

shows the spatial distributions of the fluence rates at early times $(t=20 \mathrm{ps})$ after a light pulse has been irradiated, and here, the spatial distributions are dependent on the numerical models and conditions. At late times ( $t=500 \mathrm{ps})$, the spatial distributions calculated from the DE and RTE are quite similar [Fig. 6(b)] suggesting that a spatially and temporally hybrid is possible in TD.

\subsection{Improvement of Diffuse Optical Tomography Image Quality}

The quality of DOT images depends on several factors, including the spatial resolution, image contrast, and artifacts that may be present. These factors are affected by the DOT algorithms as well as the quality of the instruments. As the inverse problem is ill-posed, a regularization term is generally incorporated, and various regularization methods have been tried. Finding an appropriate regularization parameter is crucial to the reconstruction of high-quality images. ${ }^{109,110}$ A recent cerebral DOT study has reported that the appropriate regularization parameter varies with the number of activation spots; the linearly constrained minimum variance beamforming is the best for single spot activation, while the minimum $l_{1}$-norm estimate resolves two activation spots best. ${ }^{110}$

Unlike magnetic resonance imaging (MRI) and x-ray CT, DOT itself does not provide anatomical (structural) information, which makes it difficult to solve the forward model correctly. Combining DOT and MRI is a useful approach to get around this issue. Tissue geometry obtained from segmented MR images is used to constrain the optical model, leading to improvements in the quality of reconstructed images. ${ }^{111-113}$ However, it is not always possible to obtain MRI data. When DOT is applied to neuroimaging studies, a general head atlas (the Montréal Neurological Institute template ${ }^{114}$ )-guided DOT has also been proposed. ${ }^{115,116}$ For measurements of organs/ tissue other than the brain, x-ray-guided (e.g., bone) ${ }^{117}$ and ultrasound-guided $\mathrm{DOT}^{118,119}$ (e.g., the breast and prostate) have also been reported to improve the accuracy of image reconstruction.

\section{Clinical Applications}

Linearization approaches applying DOT are exclusively used in neuroimaging studies, ${ }^{28,29,70,120}$ while various other organs and tissue including breast cancer, are measured by DOT based on the nonlinear iterative approaches. The following presents examples of clinical applications of DOT.

\subsection{Brain Imaging}

The DOT studies based on linearization approaches are typically performed by $\mathrm{CW}$ instruments, with fast sampling rates preferable to neuroimaging studies. Zeff et al. ${ }^{28}$ first succeeded in retinotopic mapping of adult human visual cortex using DOT, and then the DOT has found a wide variety of applications in brain science, such as somatosensory processing ${ }^{29}$ and speech processing. ${ }^{121}$ DOT has also been applied to investigation of infants (cerebral hemodynamic changes during face perception) $)^{122}$ and neonates (cortical hemodynamics during seizures). ${ }^{123}$ However, using TD instruments is also possible. A study with a TD system has clearly demonstrated that DOT enables detection of brain activity-related changes in the cerebral $\mathrm{Hb}$ with little contamination from hemodynamic changes in the extracerebral tissue. ${ }^{124}$ Currently, resting-state fMRI (functional MRI) is a focus of research interest, and NIRS has also been employed in investigating functional connectivity. However, the scalp blood flow fluctuates during resting, as it is also the case with the cerebral blood flow, making it necessary to distinguish NIRS signals originating in cerebral tissue from those coming from the scalp. With DOT, this issue would be solved and such investigations should be performed by DOT. ${ }^{125,126}$ Very recently, resting-state functional connectivity in infants has been investigated at the bedside by using highdensity DOT. The results have indicated that this technique is a powerful approach to understand functional cerebral development. $^{127}$

The DOT based on nonlinear iterative approaches was developed mainly aiming to reconstruct 3-D images of tissue oxygenation and blood volume in the neonatal brain at bedside, ${ }^{128,129}$ and multichannel TD systems were developed to be able to do this. ${ }^{130,131}$ Iterative approaches were also applied to functional brain mapping in neonates. ${ }^{132}$ Except with extremely low birth weight infants, where the head circumference is very small as in Fig. 4(b), light re-emitting from the human head is hardly detected at the head surface opposite to the source position. It may be concluded that DOT based on either linear or nonlinear iterative approaches rarely provides information of subcortical structures, and this is a limitation for DOT when it is applied to brain imaging.

\subsection{Breast Cancer Imaging}

Breast cancer is one of the most common cancers and screening is recommended for early detection of these cancers. Currently, the most common and effective screening technique is $\mathrm{x}$-ray mammography combined with a physical examination of the breast. Although the overall specificity of x-ray mammography in breast cancer detection is high, it presents a number of drawbacks such as radiation exposure and a relatively high probability of both false-positives and false-negatives. Here DOT has been considered a promising alternative approach, and it has been extensively studied for diagnosis and evaluation of treatment responses. ${ }^{118,133-137}$ However, the minimum reliably resolved feature size is still insufficient for clinical application 
(7 to $10 \mathrm{~mm}),{ }^{138,139}$ and new methods to improve the image quality, such as structural-prior guided approaches are needed for further progress here. ${ }^{139}$

\subsection{Muscle, Peripheral Circulation, and Joint Imaging}

In the field of exercise physiology, electromyography is a principal noninvasive assessment of skeletal muscle functions. Here NIRS has also been employed to examine oxygenation in muscles during the performance of exercises, ${ }^{140,141}$ but a quantitative evaluation and distinguishing between muscle and adipose tissue are difficult. ${ }^{142}$ Although only some research groups have reported DOT images of exercising muscles, the findings have suggested the possibility that DOT may be a useful method to examine the physiological functions of muscles. ${ }^{143-146}$

Peripheral artery disease (PAD) is a disease with narrowing of arteries caused by buildup of plaque in the vascular wall. This reduces the blood supply to the extremities and causes intermittent claudication, and finally critical ischemia. The ankle brachial index, the ratio of the maximum systolic blood pressure at the ankle to the systolic blood pressure in the brachial artery in the upper arm, is used for diagnosis and assessment of the severity of PAD. Unlike this indirect method, DOT has been employed to assess the peripheral circulation at the foot directly, in visualization of the circulation. ${ }^{147}$

A unique DOT application is imaging of rheumatoid arthritis (RA). Low-field (0.2T) MRI and ultrasound imaging can be used to establish a diagnosis of RA, while MRI is expensive and ultrasound imaging needs contact measurements, which may cause pain. DOT is a potentially attractive alternative diagnostic method. Since, however, the diffusion approximation is not valid in fingers, where the source detector distance is small, the RTE-based algorithm is required. Here frequencydomain DOT (FD DOT) imaging has demonstrated clear differences between healthy subjects and patients with RA. ${ }^{148,149}$

\subsection{Thyroid Imaging}

There are no reports of DOT imaging of the thyroid gland. This is probably because ultrasound is the primary modality in assessments of thyroid lesions. However, a differential diagnosis distinguishing between thyroid follicular cancer and follicular adenomas is extremely difficult even with fine needle aspiration, and here it is expected that DOT will be able to get around this problem. In addition, the human neck anatomy is optically interesting and challenging, with the presence of void space (the trachea). ${ }^{150}$ Here, the RTE will need to be used as the forward model, and reflection and refraction at the trachea boundary have to be considered. A recent numerical experiment by our group will be presented next (unpublished data). In this experiment, first, a realistic 3-D human neck model was developed with MRI data [Fig. 7(a)], and 23 sources and 22 detectors were placed alternately on the front surface of the neck with the arrangement of light from each source to be detected by seven detectors [Fig. 7(b)]. The model has two thyroid cancers with different $\mu_{\mathrm{a}}$ values from the $\mu_{\mathrm{a}}$ of the thyroid. Our algorithm is based on the time domain RTE and the Gauss-Newton method, and MRI data are used as the priori anatomical information. Figure 7(c) demonstrates reconstructed images of the absorption coefficient of the human neck, and the thyroid is (a)

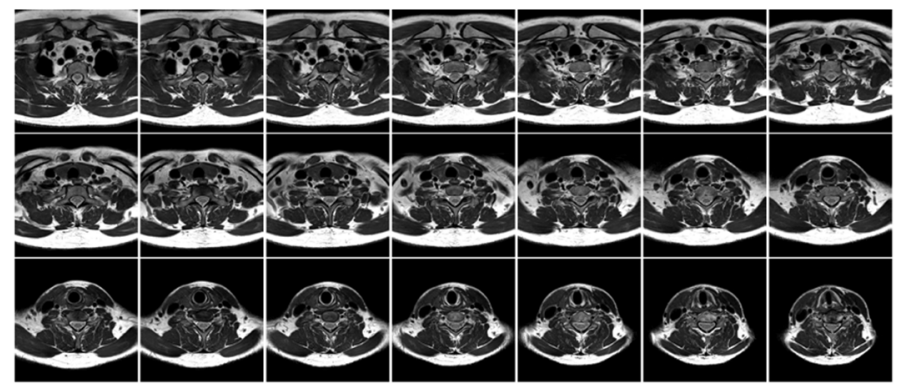

(b)

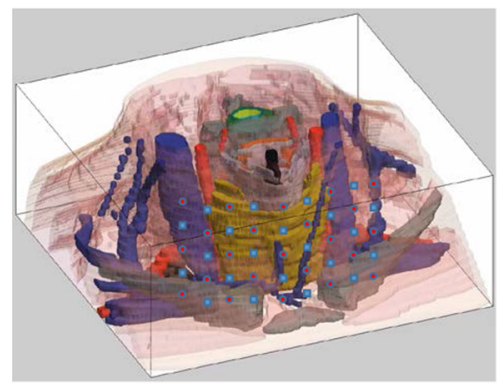

(c)
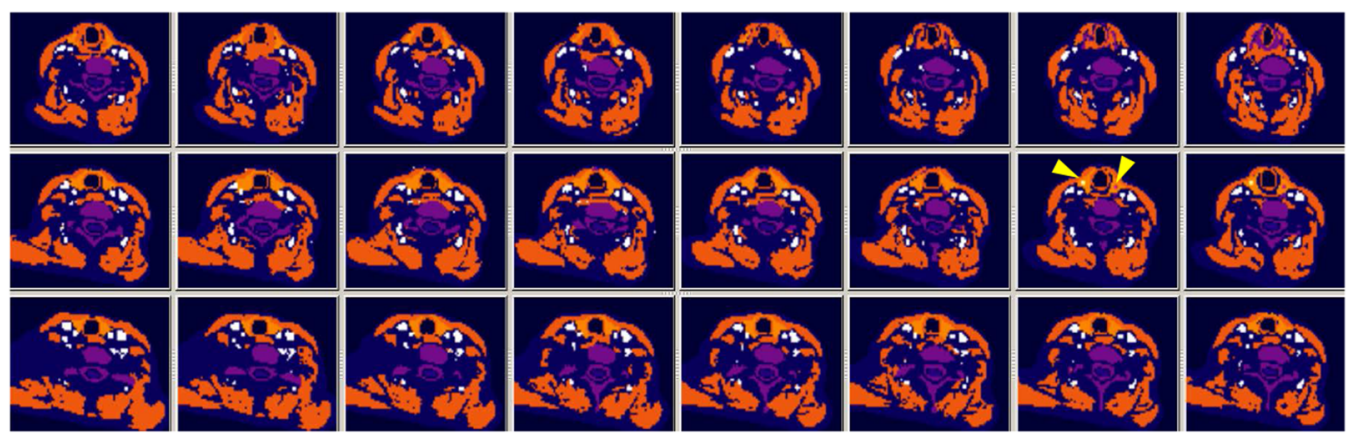

Fig. 7 RTE-based image reconstruction of the thyroid model. (a) MR images of the human neck. (b) Realistic human neck model based on the MR images. Red and blue spots denote source and detector positions, respectively. Red spots also detect light when they are not a source position. (c) Reconstructed images of $\mu_{\mathrm{a}}$ of the thyroid (white arrow). The yellow triangles denote cancers. (Reported in ISEM 2015 by K. Hashimoto et al.) 
more clearly imaged than with MRI. Two cancers are also reconstructed.

\section{Future Prospects}

Real-time image recovery, which is possible with conventional NIRS, is the aim of the development of high-density DOT based on linearization approaches. ${ }^{151}$ Very different from this, developing DOT-based nonlinear iterative approaches is far more challenging and complex. There is still room for improvements; however, several advantages in DOT over other imaging techniques stress the need for DOT applications in clinical medicine. It is able to detect lesions without exogenous probes or contrast agents, it can be employed to measure neonates, and it is useful for planning of photodynamic therapy. Furthermore, DOT can be extended to fluorescence tomography (FDOT). ${ }^{152-155}$ With FDOT, molecular imaging in living human subjects is possible. Thus, combining DOT and FDOT will make dynamic multilevel, from molecular to whole organism level, bio-optical imaging possible.

\section{Acknowledgments}

This research was supported in part by Japan Agency for Medical Research and Development and JSPS KAKENHI Grant No. 25287028.

\section{References}

1. F. F. Jöbsis, "Noninvasive infrared monitoring of cerebral and myocardial oxygen sufficiency and circulatory parameters," Science 198, 1264 1267 (1977).

2. B. Chance et al., "Cognition-activated low frequency modulation of light absorption in human brain," Proc. Natl. Acad. Sci. U. S. A. 90, 3770-3774 (1993).

3. Y. Hoshi and M. Tamura, "Detection of dynamic changes in cerebral oxygenation coupled to neuronal function during mental work in man," Neurosci. Lett. 150, 5-8 (1993).

4. T. Kato et al., "Human visual cortical function during photic stimulate on monitoring by means of near-infrared spectroscopy," J. Cereb. Blood Flow Metab. 13, 516-520 (1993).

5. A. Villringer et al., "Near-infrared spectroscopy (NIRS): a new tool to study hemodynamic changes during activation of brain function in human adults," Neurosci. Lett. 154, 101-104 (1993).

6. J. C. Hebden and K. S. Wing, "Time-resolved optical tomography," Appl. Opt. 32, 372-380 (1993).

7. S. R. Arridge, "Optical tomography in medical imaging," Inv. Prob. 15, R41-R93 (1999).

8. A. H. Hielscher et al., "Near-infrared diffuse optical tomography," Dis. Markers. 18, 313-337 (2002).

9. K. Ren, "Recent developments in numerical techniques for transportbased medical imaging methods," Commun. Comput. Phys. 8, 1-50 (2010).

10. B. B. Das, K. M. Yoo, and R. R. Alfano, "Ultrafast time-gated imaging in thick tissues: a step toward optical mammography," Opt. Lett. 18, 1092-1094 (1993).

11. L. Wang et al., "Ballistic 2-D imaging through scattering walls using an ultrafast optical Kerr gate," Science 253, 769-771 (1991).

12. G. Jarry et al., "Imaging mammalian tissues and organs using laser collimated transillumination," J. Biomed. Eng. 6, 70-74 (1984).

13. P. C. Jackson et al., "The development of a system for transillumination computed tomography," Br. J. Radiol. 60, 375-380 (1987).

14. R. Abumi et al., "Imaging of rat head under ischemic conditions by near-infrared computed tomography," J. Clin. Biochem. Nutr. 11, 211-222 (1991).

15. I. R. Singer et al., "Image reconstruction of the interior of bodies that diffusion radiation," Science 248, 990-993 (1990).

16. S. R. Arridge, M. Schweiger, and D. T. Delpy, "Iterative reconstruction of near infra-red absorption images," Proc. SPIE 1767, 372-383 (1992).
17. R. L. Barbour et al., "Imaging of diffusing media by a progressive iterative backprojection method using time-domain data," Proc. SPIE 1641, 21-34 (1992).

18. B. W. Pogue et al., "Initial assessment of s simple system for frequency domain diffuse optical tomography," Phys. Med. Biol. 40, 1709-1729 (1995).

19. J. C. Hebden, S. R. Arridge, and D. T. Delpy, "Optical imaging in medicine: II. Modelling and reconstruction," Phys. Med. Biol. 42, 841 (1997).

20. S. R. Arridge and J. C. Schotland, "Optical tomography: forward and inverse problems," Inv. Prob. 25, 123010 (2009).

21. G. Gratton et al., "Shades of gray matter: noninvasive optical images of human brain responses during visual stimulation," Psychophysiology 32, 505-509 (1995).

22. A. Maki et al., "Spatial and temporal analysis of human motor activity using noninvasive NIR topography," Med. Phys. 22, 1997-2005 (1995).

23. T. Yamamoto et al., "Arranging optical fibres for the spatial resolution improvement of topographical images," Phys. Med. Biol. 47, 34293440 (2002).

24. H. Kawaguchi et al., "Theoretical evaluation of accuracy in position and size of brain activity obtained by near-infrared topography," Phys. Med. Biol. 49, 2753-2765 (2004).

25. S. Wang et al., "Effects of spatial variation of skull and cerebrospinal fluid layers on optical mapping of brain activities," Opt. Rev. 17, 410 420 (2010).

26. T. Takahashi et al., "Influence of skin blood flow on near-infrared spectroscopy signals measured on the forehead during a verbal fluency task," NeuroImage 57, 991-1002 (2011).

27. E. Kirillina et al., "The physiological origin of task-evoked systemic artefacts in functional near infrared spectroscopy," NeuroImage 61, 70-81 (2012).

28. B. W. Zeff et al., "Retinotopic mapping of adult human visual cortex with high-density diffuse optical tomography," Proc. Natl. Acad. Sci. U. S. A. 104, 12169-12174 (2007).

29. C. Habermehl et al., "Somatosensory activation of two fingers can be discriminated with ultrahigh-density diffuse optical tomography," NeuroImage 59, 3201-3211 (2012).

30. T. Correia et al., "Three-dimensional optical topography of brain activity in infants watching videos of human movement," Phys. Med. Biol. 57, 1135-1146 (2012).

31. A. M. Smith, M. C. Mancini, and S. Nie, "Second window for in vivo imaging," Nat. Nanotechnol. 4, 710-711 (2009).

32. T. Hamaoka et al., "The use of muscle near-infrared spectroscopy in sports, health and medical sciences: recent developments," Philos. Trans. R. Soc. A 369, 4591-4604 (2011).

33. M. Ferrari, M. Muthalib, and V. Quaresima, "The use of muscle nearinfrared spectroscopy in understanding skeletal muscle physiology: recent developments," Philos. Trans. R. Soc. A 369, 4577-4590 (2011).

34. Y. Hoshi et al., "Redox behavior of cytochrome oxidase in the rat brain measured by near-infrared spectroscopy," J. Appl. Physiol. 83, 18421848 (1997).

35. G. Bale et al., "A new broadband near-infrared spectroscopy system for in-vivo measurements of cerebral cytochrome-c-oxidase changes in neonatal brain injury," Biomed. Opt. Express 5(10), 3450-3466 (2014).

36. M. Ferrari and V. Quaresima, "A brief review on the history of human functional near-infrared spectroscopy (fNIRS) development and fields of application," NeuroImage 63(2), 921-935 (2012).

37. S. L. Jacques, C. A. Alter, and S. A. Prahl, "Angular dependence of HeNe laser light scattering by human dermis," Lasers Life Sci. 1(4), 309-334 (1987).

38. S. T. Flock, B. C. Wilson, and M. S. Patterson, "Total attenuation coefficients and scattering phase functions of tissues and phantom materials at 633 nm," Med. Phys. 14(5), 835-843 (1987).

39. M. S. Patterson, B. C. Wilson, and D. R. Wyman, "The propagation of optical radiation in tissue I. Models of radiation transport and their application," Lasers Med. Sci. 6, 155-168 (1991).

40. A. Kienle et al., "Spatially resolved absolute diffuse reflectance measurements for noninvasive determination of the optical scattering and absorption coefficients of biological tissue," Appl. Opt. 35(13), 2304-2314 (1996).

41. J. J. Duderstadt and W. R. Martin, Transport Theory, John Wiley \& Sons, New York (1979). 
42. A. Ishimaru, Wave Propagation and Scattering in Random Media, IEEE Press, New York (1997).

43. P. Wang, S. R. Arridge, and M. Jiang, "Radiative transfer equation for media with spatially varying refractive index," Phys. Rev. A 90, 023803 (2014).

44. A. D. Klose, "The forward and inverse problem in tissue optics based on the radiative transfer equation: a brief review," J. Quant. Spectrosc. Radiat. Transfer 111, 1852-1853 (2010).

45. L. G. Henyey and L. J. Greenstein, "Diffuse radiation in the galaxy," J. Astrophys. 93, 70-83 (1941).

46. K. Furutsu and Y. Yamada, "Diffusion approximation for a dissipative random medium and the applications," Phys. Rev. E 50(5), 3634-3640 (1994).

47. R. Pierrat, J.-J. Greffet, and R. Carminati, "Photon diffusion coefficient in scattering and absorbing media," J. Opt. Soc. Am. A Opt. Imag. Sci. Vis. 23, 1106-1110 (2006).

48. D. T. Delpy et al., "Estimation of optical pathlength through tissue from direct time of flight measurement," Phys. Med. Biol. 33, 1433-1442 (1988).

49. B. Chance et al., "Comparison of time-resolved and -unresolved measurements of deoxyhemoglobin in brain," Proc. Natl. Acad. Sci. U. S. A. 85, 4971-4975 (1988).

50. J. R. Lakowicz and K. Berndt, "Frequency domain measurement of photon migration in tissues," Chem. Phys. Lett. 166, 246-252 (1990).

51. A. Duncan et al., "A multiwavelength, wideband, intensity modulated optical spectrometer for near infrared spectroscopy and imaging," Proc. SPIE 1888, 248-257 (1993).

52. J. Steinbrink et al., "Determining changes in NIR absorption using a layered model of the human head," Phys. Med. Biol. 46, 879-896 (2001).

53. A. Liebert et al., "Time-resolved multidistance near-infrared spectroscopy of the adult head: intracerebral and extracerebral absorption changes from moments of distribution of times of flight of photons," Appl. Opt. 43, 3037-3047 (2004).

54. M. S. Patterson, B. Chance, and B. C. Wilson, "Time resolved reflectance and transmittance for the non-invasive measurement of tissue optical properties," Appl. Opt. 28(12), 2331-2336 (1989).

55. A. Torricelli et al., "Time domain functional NIRS imaging for human brain mapping," Neurolmage 85, 28-50 (2014).

56. C. Sato et al., "Extraction of depth-dependent signals from timeresolved reflectance in layered turbid media," J. Biomed. Opt. 10, 064008 (2005).

57. C. Sato et al., "Intraoperative monitoring of depth-dependent hemoglobin concentration changes during carotid endarterectomy by timeresolved spectroscopy," Appl. Opt. 46, 2785-2792 (2007).

58. B. Chance et al., "Time-resolved spectroscopy of hemoglobin and myoglobin in resting and ischemic muscle," Anal. Biochem. 174, 698-707 (1988).

59. A. H. Hielscher et al., "Near-infrared diffuse optical tomography," Dis. Markers 18(5-6), 313-337 (2002).

60. S. R. Arridge, M. Cope, and D.T. Delpy, "Theoretical basis for the determination of optical pathlengths in tissue: temporal and frequency analysis," Phys. Med. Biol. 37, 1531-1560 (1992).

61. J. B. Fishkin and E. Graton, "Propagation of photon-density waves in strongly scattering media containing an absorbing semi-infinite plane bounded by a straight edge," J. Opt. Soc. Am. A. 10(1), 127-140 (1993).

62. S. Fantini, M. A. Franceschini, and E. Graton, "Semi-infinite geometry boundary problem for light migration in highly scattering media: a frequency-domain study in the diffusion approximation," J. Opt. Soc. Am. B 11, 2128-2138 (1994).

63. S. Fantini et al., "Quantitative determination of the absorption spectra of chromophores in strongly scattering media: a light-emitting-diode based technique," Appl. Opt. 33(22), 5204-5213 (1994).

64. V. Toronov et al., "Study of local cerebral hemodynamics by frequencydomain near-infrared spectroscopy and correlation with simultaneously acquired functional magnetic resonance imaging," Opt. Express 9(8), 417-427 (2001).

65. A. Kienle and M. S. Patterson, "Improved solutions of the steadystate and the time-resolved diffusion equations for reflectance from a semi-infinite turbid medium," J. Opt. Soc. Am. A 14(1), 246-254 (1997).
66. M. A. O'Leary et al., "Experimental images of heterogenous turbid media by frequency-domain diffusing-photon tomography," Opt. Lett. 20, 426-428 (1995).

67. R. L. Barbour et al., "Perturbation approach for optical diffusion tomography using continuous-wave and time-resolved data," Medical Optical Tomography: Functional Imaging and Monitoring, G. Muller et al., Eds., Vol. IS11, pp. 87-120, SPIE Press, Bellingham (1993).

68. D. A. Boas, "A fundamental limitation of linearized algorithms for diffuse optical tomography," Opt. Express 1(13), 404-413 (1997).

69. N. M. Gregg et al., "Brain specificity of diffuse optical imaging: improvements from superficial signal regression and tomography," Front. Neuroenerg. 2, 14 (2010).

70. A. T. Eggebrecht et al., "Mapping distributed brain function and networks with diffuse optical tomography," Nat. Photonics 8(6), 448-454 (2014).

71. D. A. Boas, A. M. Dale, and M. A. Franceshini, "Diffuse optical imaging of brain activation: approaches to optimizing image sensitivity, resolution, and accuracy," NeuroImage 23, S275-S288 (2004).

72. T. Shimokawa et al., "Hierarchical Bayesian estimation improved depth accuracy and spatial resolution of diffuse optical tomography," Opt. Express 20(18), 20427-20446 (2012).

73. B. W. Pogue et al., "Spatially variant regularization improve diffuse optical tomography," Appl. Opt. 38, 2950-2961 (1999).

74. J. P. Culver et al., "Diffuse optical tomography of cerebral blood flow, oxygenation, and metabolism in rat during focal ischemia," J. Cereb. Blood Flow Metab. 23, 911-924 (2003).

75. N. Cao, A. Nehorai, and M. Jacob, "Image reconstruction for diffuse optical tomography using sparsity regularization and expectation-maximization algorithm," Opt. Express 15(21), 13695-13708 (2007).

76. M. Jacob et al., "Level-set algorithm for the reconstruction of functional activation in near-infrared spectroscopic imaging," J. Biomed. Opt. 11(6), 064029 (2006).

77. M. Guven et al., "Diffuse optical tomography with a priori anatomical information," Phys. Med. Boil. 50, 2837 (2005).

78. F. Abdelnour, C. Genovese, and T. Huppert, "Hierarchical Bayesian regularization of reconstructions for diffuse optical tomography using multiple priors," Biomed. Opt. Express 1(4), 1084-1103 (2010).

79. T. Shimokawa et al., "Extended hierarchical Bayesian diffuse optical tomography for removing scalp artifact," Biomed. Opt. Express 4(11), 2411-2432 (2013).

80. O. Lee et al., "Compressive diffuse optical tomography: noniterative exact reconstruction using joint sparsity," IEEE Trans. Med. Imaging 30(5), 1129-1142 (2011).

81. O. Lee and J. C. Ye, "Joint sparsity-driven non-iterative simultaneous reconstruction of absorption and scattering in diffuse optical tomography," Opt. Express 21(22), 26589-26604 (2013).

82. S. Saquib, K. M. Hanson, and G. S. Cunningham, "Model-based image reconstruction from time-resolved diffusion data," Proc. SPIE 3034, 369-380 (1997).

83. A. H. Hielscher, "Model-based iterative image reconstruction for photon migration tomography," Proc. SPIE 3171, 106-117 (1997).

84. S. R. Arridge and M. Schweiger, "Photon-measurement density functions. Part 2: finite-element-method calculation," Appl. Opt. 34(34), 8026-8037 (1995).

85. K. D. Paulsen and H. Jiang, "Spatially varying optical property reconstruction using a finite element diffusion equation approximation," Med. Phys. 22(6), 691-701 (1995).

86. S. A. Arridge and M. Schweiger, "A gradient-based optimization scheme for optical tomography," Opt. Express 2(6), 213-226 (1998).

87. A. H. Hielscher, A.D. Klose, and K. M. Hanson, "Gradient-based iterative image reconstruction scheme for time-resolved optical tomography," IEEE Trans. Med. Imaging 18(3), 262-271 (1999).

88. M. Schweiger and S. R. Arridge, "Application of temporal filters to time resolved data in optical tomography," Phys. Med. Biol. 44, 1699 (1999).

89. F. Gao, H. Zhao, and Y. Yamada, "Improvement of image quality in diffuse optical tomography by use of full time-resolved data," Appl. Opt. 41(4), 778-791 (2002).

90. F. A. Grünbaum, "A nonlinear inverse problem inspired by three-dimensional diffuse tomography," Inverse Probl. 17, 1907-1922 (2001).

91. H. Dehghani et al., "Numerical modeling and image reconstruction in diffuse optical tomography," Philos. Trans. R. Soc. A 367, 3073-3093 (2009). 
92. A. H. Hielscher, R. E. Alcouff, and R. L. Barbour, "Comparison of finite-difference transport and diffusion calculation for photon migration in homogeneous and heterogeneous tissues," Phys. Med. Biol. 43, 1285-1302 (1998).

93. H. Fujii et al., "Hybrid model of light propagation in random media based on the time-dependent radiative transfer," J. Quant. Spectrosc. Radiat. Transfer 147, 145-154 (2014).

94. A. D. Klose and A. H. Hielscher, "Optical tomography with the equation of radiative transfer," Int. J. Numer. Methods Heat Fluid Flow 18(3/4), 443-464 (2008).

95. A. Charette, J. Boulanger, and H. K. Kim, "An overview on recent radiation transport algorithm development for optical tomography imaging," J. Quant. Spectrosc. Radiat. Transfer 109, 2743-2766 (2008).

96. A. D. Klose and A. H. Hielscher, "Iterative reconstruction scheme for optical tomography based on the equation of radiative transfer," Med. Phys. 26(8), 1698-1707 (1999).

97. C. Das et al., "Experimental and numerical analysis of short-pulse laser interaction with tissue phantoms containing inhomogeneities," Appl. Opt. 42(25), 5173-5180 (2003).

98. J. Boulanger and A. Charette, "Numerical developments for shortpulsed near infra-red laser spectroscopy. Part I: direct treatment," J. Quant. Spectrosc. Radiat. Transfer 91, 189-209 (2005).

99. M. Machida et al., "The Green's function for the radiative transport equation in the slab geometry," J. Phys. A: Math. Theor. 43, 065402 (2010).

100. A. Liemert and A. Kienle, "Exact and efficient solution of the radiative transport equation for the semi-infinite medium," Sci. Rep. 3, 2018 (2013).

101. Z. Guo and K. Kim, "Ultrafast-laser-radiation transfer in heterogeneous tissues with the discrete-ordinate method," Appl. Opt. 42(16), 2897-2905 (2003).

102. T. Tarvainen et al., "Hybrid radiative-transfer-diffusion model for optical tomography," Appl. Opt. 44(6), 876-886 (2005).

103. H. Gao and H. Zhao, "A fast-forward solver of radiative transfer equation," Transp. Theory Stat. Phys. 38(3), 149-192 (2009).

104. P. González-Rodríguez and A. D. Kim, "Comparison of light scattering models for diffuse optical tomography," Opt. Express 17(11), 8756-8774 (2009).

105. J. Jia, H. K. Kim, and A. H. Hielscher, "Fast linear solver for radiative transport equation with multiple right hand sides in diffuse optical tomography," J. Quant. Spectrosc. Radiat. Transfer 167, 10-22 (2015).

106. T. Tarvainen et al., "Coupled radiative transfer equation and diffusion approximation model for photon migration in turbid medium with low-scattering and non-scattering regions," Phys. Med. Biol. 50, 4913-4930 (2005).

107. T. Tarvainen et al., "Image reconstruction in diffuse optical tomography using the coupled radiative transport-diffusion model," J. Quant. Spectrosc. Radiat. Transfer 112, 2600-2608 (2011).

108. H. Fujii et al., "A coupling model of the radiative transport equation for calculating photon migration in biological tissue," Proc. SPIE 9792, 979214 (2015).

109. T. Correria et al., "Selection of regularization parameter for optical topography," J. Biomed. Opt. 14(3), 034044 (2009).

110. C. Habermehl et al., "Optimizing the regularization for imaginf reconstruction of cerebral diffuse optical tomography," J. Biomed. Opt. 19(9), 096006 (2014).

111. A. H. Barnett et al., "Robust inference of baseline optical properties of the human head with three-dimensional segmentation from magnetic resonance imaging," Appl. Opt. 42(16), 3095-3108 (2003).

112. P. K. Yalavarthy et al., "Structural information within regularization matrices improves near infrared diffuse optical tomography," Opt. Express 15(13), 8043-8058 (2007).

113. L. Zhang et al., "Direct regularization from co-registered anatomical images for MRI-guided near-infrared spectral tomographic image reconstruction," Biomed. Opt. Express 6(9), 3618-3630 (2015).

114. D. L. Collins et al., "Design and construction of a realistic digital brain phantom," IEEE Trans. Med. Imaging 17(3), 463-468 (1998).

115. A. Custo et al., "Anatomical atlas-guided diffuse optical tomography of brain activation," Neurolmage 49(1), 561-567 (2010).

116. Z. Lin et al., "Atlas-guided volumetric diffuse optical tomography enhanced by generalized linear model analysis to image risk decision-making responses in young adults," Hum. Brain Mapp. 35, 4249-4266 (2014).

117. Z. Yuan et al., "Tomographic x-ray-guided three-dimensional diffuse optical tomography of osteoarthritis in the finger joints," J. Biomed. Opt. 13(4), 044006 (2008).

118. C. Xu et al., "Ultrasound-guided diffuse optical tomography for predicting and monitoring neoadjuvant chemotherapy of breast cancers: recent progress," Ultrason. Imaging 38(1), 5-18 (2015).

119. V. C. Kavuri and H. Liu, "Hierarchical clustering method to improve transrectal ultrasound-guided diffuse optical tomography for prostate cancer imaging," Acad. Radiol. 21(2), 250-262 (2014).

120. T. Correia et al., "Three-dimensional optical topography of brain activity in infants watching videos of human movement," Phys. Med. Biol. 57, 1135-1146 (2012).

121. M. S. Hassanpour et al., "Mapping cortical responses to speech using high-density diffuse optical tomography," NeuroImage 117, 319-326 (2015).

122. A. Blasi et al., "Investigation of depth dependent changes in cerebral haemodynamics during face perception in infants," Phys. Med. Biol. 52, 6849-6864 (2007).

123. H. Singh et al., "Mapping cortical haemodynamics during neonatal seizures using diffuse optical tomography: a case study," Neurolmage: Clin. 5, 256-265 (2014).

124. Y. Ueda et al., "Reflectance diffuse optical tomography; its application to human brain mapping," Jpn. J. Appl. Phys. 44(38), L1203-L1206 (2005).

125. B. R. White et al., "Resting-state functional connectivity in the human brain revealed with diffuse optical tomography," NeuroImage 47, 148156 (2009).

126. B. R. White et al., "Bedside optical imaging of occipital resting-state functional connectivity in neonates," NeuroImage 59, 2529-2538 (2012).

127. S. L. Ferradal et al., "Functional imaging of the developing brain at the bedside using diffuse optical tomography," Cereb. Cortex 26, 1558-1568 (2016).

128. J. C. Hebden et al., "Imaging changes in blood volume and oxygenation in the newborn infant brain using three-dimensional optical tomography," Phys. Med. Biol. 49, 1117 (2004).

129. R. Fukuzawa, "Diffuse optical tomography for premature neonate head," Master's Thesis, The University of Electro-Communications, Tokyo, Japan (2009).

130. H. Eda et al., "Multichannel time-resolved optical tomographic imaging system," Rev. Sci. Instrum. 70(9), 3595-3602 (1999).

131. F. E. W. Schmidt et al., "A 32-channel time-resolved instrument for medical optical tomography," Rev. Sci. Instrum. 71(1), 256-265 (2000).

132. A. P. Gibson et al., "Three-dimensional whole-head optical tomography of passive motor evoked responses in the neonate," NeuroImage 30, 521-528 (2006).

133. P. Taroni et al., "Time-resolved optical mammography between 637 and $985 \mathrm{~nm}$ : clinical study on the detection and identification of breast lesions," Phys. Med. Biol. 50(11), 2469-2488 (2005).

134. L. C. Enfield et al., "Three-dimensional time-resolved optical mammography of the uncompressed breast," Appl. Opt. 46(17), 36283638 (2007).

135. A. Cerussi et al., "Predicting response to breast cancer neoadjuvant chemotherapy using diffuse optical spectroscopy," Proc. Natl. Acad. Sci. U. S. A. 104(10), 4014-4019 (2007).

136. R. Choe et al., "Differentiation of benign and malignant breast tumors by in-vivo three-dimensional parallel-plate diffuse optical tomography," J. Biomed. Opt. 14(2), 024020 (2009).

137. M. L. Flexman et al., "Monitoring early response to drug therapy with diffuse optical tomography," J. Biomed. Opt. 17(1), 016014 (2012).

138. S. D. Konecky et al., "Imaging complex structures with diffuse light," Opt. Express 16(7), 5048-5060 (2008).

139. B. Deng et al., "Characterization of structural-prior guide optical tomography using realistic breast models derived from dual-energy x-ray mammography," Biomed. Opt. Express 6(7), 2366-2379 (2015).

140. D. S. DeLorey, J. M. Kowalchuk, and D. H. Paterson, "Relationship between pulmonary $\mathrm{O}_{2}$ uptake kinetics and muscle deoxygenation during moderate-intensity exercise," J. Appl. Physiol. 95(1), 113-120 (2003). 
141. M. Niwayama et al., "A 200-channel imaging system of muscle oxygenation using $\mathrm{CW}$ near-infrared spectroscopy," IEICE Trans. Inf. Syst. E85-D(1), 115-123 (2002).

142. E. Omae et al., "Sensitivity correction for the influence of the fat layer on muscle oxygenation and estimation of fat thickness by timeresolved spectroscopy," J. Biomed. Opt. 19(6), 067005 (2014).

143. L. S. L. Arakaki et al., "Optical diffusion tomograpohy of the exercising human forearm," in Biomedical Optical Spectroscopy and Diagnostics, OSA Trends in Optics and Photonics, Optical Society of America (2000).

144. E. M. C. Hillman et al., "Time resolved optical tomography of the human forearm," Phys. Med. Biol. 46, 1117-1130 (2001).

145. H. Zhao et al., "Time-resolved diffuse optical tomographic imaging for the provision of both anatomical and functional information about biological tissue," Appl. Opt. 44(1), 1905-1916 (2005).

146. Y. Tanikawa et al., "Time-resolved DOT images of human forearm under exercise," in Proc. APBP 2009, pp. 120-121 (2009).

147. M. A. Khali et al., "Dynamic diffuse optical imaging of peripheral arterial disease," Biomed. Opt. Express 3(9), 2288-2298 (2012).

148. L. D. Montejo et al., "Computer-aided diagnosis of rheumatoid arthritis with optical tomography, Part 1: feature extraction," J. Biomed. Opt. 18(7), 076001 (2013).

149. L. D. Montejo et al., "Computer-aided diagnosis of rheumatoid arthritis with optical tomography, Part 2: image classification," J. Biomed. Opt. 18(7), 076002 (2013).

150. H. Fujii et al., "Numerical modeling of photon migration in human neck based on the radiative transport equation," J. Appl. Nonlinear Dyn. 5(1), 117-125 (2016).

151. W. Wu et al., "Fast and efficient image reconstruction for high density diffuse optical imaging of the human brain," Biomed. Opt. Express 6(11), 4567-4584 (2015).
152. V. Nitzachristos et al., "Fluorescence molecular tomography resolves protease activity in vivo," Nat. Med. 8(7), 757-761 (2002).

153. A. Corlu et al., "Three-dimensional in vivo fluorescence diffuse optical tomography of breast cancer in humans," Opt. Express 15(11), 66966716 (2007).

154. A. Marjono et al., "Total light approach of time-domain fluorescence diffuse optical tomography," Opt. Express 16(19), 15268-15285 (2008).

155. N. Durcros et al., "Fluorescence diffuse optical tomography: timeresolved versus continuous-wave in the reflectance configuration," IRBM 32, 243-250 (2011).

Yoko Hoshi graduated from Akita University School of Medicine (MD) in 1981 and got her PhD from Hokkaido University in 1990. She is a pediatrician (a child neurologist) while she has also been participating in developing NIRS and research in cognitive neuroscience. She has been a professor in the Department of Biomedical Optics at Hamamatsu University School of Medicine since April 2015. Her recent research interest is developing diffuse optical tomography.

Yukio Yamada received his BS, MS, and PhD degrees from Tokyo Institute of Technology, Department of Mechanical Engineering. From 1974 to 2001, he worked in the Mechanical Engineering Laboratory, Agency of Industrial and Science Technology, the Japanese government. From 2001 to 2013, he was a professor in the Department of Mechanical and Intellectual Engineering, University of ElectroCommunications, Tokyo, Japan. He has been a professor emeritus at the University of Electro-Communications since 2013. He specializes in biomedical photonics and biomedical thermal engineering 